\title{
Site-selective investigation of site symmetry and site occupation of iron in Fe-doped lithium niobate crystals
}

\author{
Tonya Vitova, ${ }^{1, a)}$ Josef Hormes, ${ }^{1}$ Matthias Falk, ${ }^{2}$ and Karsten Buse ${ }^{2}$ \\ ${ }^{1}$ Institute of Physics, University of Bonn, Nußallee 12, D-53115 Bonn, Germany \\ ${ }^{2}$ Institute of Physics, University of Bonn, Wegelerstr. 8, D-53115 Bonn, Germany
}

(Received 3 December 2007; accepted 15 November 2008; published online 9 January 2009)

\begin{abstract}
Lithium niobate (LN) crystals, in particular after strong oxidation, are of great relevance for applications in photorefraction and nonlinear optics. Crystals doped with 2 or 4 wt \% of iron are studied by extended x-ray absorption fine structure spectroscopy. The Fe atoms are observed to be sixfold coordinated by oxygen atoms and incorporated into the Li site. No evidence for formation of iron oxide clusters is found. In situ x-ray absorption spectroscopy measurements of the thermoelectric oxidation of a LN:Fe $2 \mathrm{wt} \%$ crystal and the analyses of the partially site-selective $\mathrm{x}$-ray absorption near edge structure spectra of a LN:Fe 4 wt \% crystal provide site-selective structural information. We found that the $\mathrm{Fe}^{2+}$ and $\mathrm{Fe}^{3+}$ sites have the same site symmetries and positions in the $\mathrm{LN}$ matrix, i.e., the $\mathrm{Fe}^{2+}$ and $\mathrm{Fe}^{3+}$ atoms are both octahedrally coordinated by six oxygen atoms and both occupy the Li site. (C) 2009 American Institute of Physics.
\end{abstract}

[DOI: $10.1063 / 1.3056116]$

\section{INTRODUCTION}

Lithium niobate $(\mathrm{LN})\left(\mathrm{LiNbO}_{3}\right)$ crystals are an important photorefractive material for applications in nonlinear optics or for holographic data storage. ${ }^{1,2}$ In order to increase the refractive index changes, leading to an enhanced storage capacity of holograms, they are doped with transition metals, e.g., $\mathrm{Fe}, \mathrm{Mn}$, or $\mathrm{Cu}$. In the case of Fe-doped LN crystals the iron atoms are found in the valence states $\mathrm{Fe}^{2+}$ and $\mathrm{Fe}^{3+}$. Upon inhomogeneous illumination, the electrons from the $\mathrm{Fe}^{2+}$ impurity centers are excited into the conduction band. They migrate due to drift, diffusion, and bulk photovoltaic effects, ${ }^{4}$ and they are finally trapped by $\mathrm{Fe}^{3+}$ centers preferentially in the dark areas. The redistributed charge builds up space-charge fields, which modulate the refractive index via the electro-optic effect.

Although light-induced refractive index changes are favorable for some applications, they are undesirable for others and called "optical damage." Some ppm of iron always exist in LN crystals. Upon illumination, refractive patterns develop, which disturb the phase matching conditions and the beam profile in nonlinear-optical applications. One way to avoid the optical damage in a LN crystal is to oxidize it. Recently, a thermoelectric method for very efficient oxidation of $\mathrm{Fe}^{2+}$ to $\mathrm{Fe}^{3+}$ in $\mathrm{LN}: \mathrm{Fe}$ crystals has been reported. ${ }^{5,6}$

Precise knowledge about the oxidation state and site occupation of the iron atoms is essential for tuning the photorefractive properties of the $\mathrm{LN}$ crystals. A recent $\mathrm{x}$-ray absorption spectroscopy (XAS) study reported the presence of $\mathrm{Fe}^{2+}$ and $\mathrm{Fe}^{3+}$ atoms in iron-doped LN crystals and their incorporation into the $\mathrm{Li}$ site. ${ }^{7}$ However, no site-selective structural information was provided, which would allow to differentiate between the $\mathrm{Fe}^{2+}$ and $\mathrm{Fe}^{3+}$ sites.

The goals of the study presented here are: to determine the incorporation site of the Fe atoms in LN crystals doped

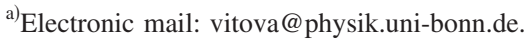

with 2 and 4 wt $\%$ of $\mathrm{Fe}$ (LN:Fe 2 wt \%, LN:Fe 4 wt \%) by the extended $\mathrm{x}$-ray absorption fine structure (EXAFS) technique, to verify that $\mathrm{Fe}^{2+}$ and $\mathrm{Fe}^{3+}$ are present in these $\mathrm{LN}$ crystals by analyzing x-ray absorption near edge structure (XANES) and visible (VIS) absorption spectra, and to provide site-selective information about the local symmetry and incorporation site of the $\mathrm{Fe}^{2+}$ and $\mathrm{Fe}^{3+}$ atoms by in situ XAS measurements of thermoelectric oxidation of a $\mathrm{LiNbO}_{3}: \mathrm{Fe} 2$ wt $\%$ crystal, nonresonant high-resolution x-ray emission (HRXE) spectroscopic measurements, and analyses of partially site-selective XANES spectra extracted from a resonant inelastic X-ray scattering (RIXS) map.

\section{SAMPLES}

The studied samples are iron-doped $\mathrm{LiNbO}_{3}$ crystals supplied by Crystal Technology, Inc. They were grown by the Czochralski technique from a congruent melt that contained 2 or 4 wt $\%$ iron oxide $\left(\mathrm{Fe}_{2} \mathrm{O}_{3}\right)$. The $\mathrm{LiNbO}_{3}: \mathrm{Fe} 2$ wt $\%$ and $\mathrm{LiNbO}_{3}: \mathrm{Fe} 4 \mathrm{wt} \%$ crystals are notated as $\mathrm{C} 1$ and C2 crystals, respectively.

\section{EXPERIMENTAL DETAILS}

XAS experiments were performed at the INE-Beamline at the ANKA $2.5 \mathrm{GeV}$ synchrotron radiation facility, Karlsruhe, Germany. For details about the instrumentation at this beamline, see Ref. 8. For energy monochromatization, a Lemonnier-type ${ }^{9}$ double crystal monochromator equipped with a set of $\mathrm{Si}(111)$ crystals was employed. The iron-doped LN crystals were ground, and absorption spectra of the powder samples and the $\mathrm{FeO}$ and $\mathrm{Fe}_{2} \mathrm{O}_{3}$ references were taken at the Fe $K$ edge in fluorescence and transmission modes, respectively. For the measurements in fluorescence mode a five-element Ge solid state detector (Canberra type) was used. During the measurements each sample was positioned 
on a goniometer; this was rotated $0.001^{\circ}$ after each spectrum with respect to the incoming beam, in order to average out the possible diffraction peaks.

All Fe $K$-edge XANES spectra were measured in the energy interval from 7060 to $7260 \mathrm{eV}$, with a $0.5 \mathrm{eV}$ step width and $2 \mathrm{~s}$ (in fluorescence mode) or $1 \mathrm{~s}$ (in transmission mode) integration time. For the EXAFS region equidistant increment was chosen in $k$ space, with $0.03 \AA^{-1}$ step width. At least six XAS (XANES and EXAFS) spectra were collected from the LN:Fe 2 wt \% and LN:Fe 4 wt \% crystals, in order to check for reproducibility of the spectral features and to improve the counting statistics. For energy calibration, the first inflection point of the $K$-edge spectrum of an iron foil measured simultaneously was set to the photon energy of $7112 \mathrm{eV}$. The data reduction in the Fe $K$-edge XANES spectra consisted of subtraction of a linear background fit to the pre-edge region from 7060 to $7100 \mathrm{eV}$ and normalization of the spectra to an edge jump of one at $7260 \mathrm{eV}$.

During the in situ XAS measurements of the thermoelectric oxidation of a $\mathrm{LN}: \mathrm{Fe} 2 \mathrm{wt} \%$ crystal, the monochromator was operated in a quick scanning mode, with 1 min measuring time per EXAFS spectrum. The LN crystal was mounted under $45^{\circ}$ with respect to the incoming photon beam. The crystal was put in a furnace in the middle of two perpendicularly crossed quartz tubes. The first tube was directed toward the fluorescence detector. The second tube provided access to the sample for the primary beam. In order to oxidize the crystal thermoelectrically, an electric field was initially applied, where the voltage and the current were restricted to $1000 \mathrm{~V}$ and $0.1 \mathrm{~mA}$, respectively. Then the crystal was heated to $700{ }^{\circ} \mathrm{C}$ with a ramp of $3.9^{\circ} \mathrm{C} / \mathrm{min}$ and abruptly cooled down. At room temperature the electric field was switched off. ${ }^{5}$

The measurements of the $\mathrm{Fe} K \beta_{1,3}$ and $\mathrm{Fe} K \beta^{\prime}$ emission lines of $\mathrm{FeO}$ and $\mathrm{Fe}_{2} \mathrm{O}_{3}$ references and the RIXS map of the $\mathrm{LN}: \mathrm{Fe} 4$ wt \% crystal were performed at the wiggler beamline W1 at HASYLAB (Hamburg). For the HRXE measurements, the incident $x$ rays were monochromatized by two $\mathrm{Si}(111)$ crystals to the energy of $7500 \mathrm{eV}$, which is approximately $400 \mathrm{eV}$ above the $\mathrm{Fe} K$ edge. $\mathrm{X}$ rays scattered from the sample were analyzed by a Johann spectrometer in dispersive geometry, ${ }^{10}$ where for the HRXE measurements and the RIXS measurement the sample was moved by 200 and $100 \mathrm{~mm}$, respectively, inside the Rowald circle. A spherically bent $\mathrm{Si}(531)$ crystal with radius of curvature of $1 \mathrm{~m}$ was employed as an analyzer and a charge coupled device camera (Roper Scientific/Princeton Instruments type) as a positionsensitive detector. The measurements were performed at a Bragg angle $\theta_{B}=71.7^{\circ}$. The experimental energy resolution is estimated as $1.4 \mathrm{eV}$ at the $\mathrm{Fe} K$ edge $(7112 \mathrm{eV})$.

The ARTEMIS (Ref. 11) program package was used for EXAFS analyses. The ionization energy $\left(E_{0}\right)$ was chosen as the energy position of the first inflection point of the respective spectrum, i.e., $7124 \mathrm{eV}$. The XAFS signals $[\chi(k)]$ measured at the $\mathrm{Fe} K$ edge, covering a $k$ range from 2.4 to $8.4 \AA^{-1}$, was Fourier transformed (FT) to $R$ space using a $k$ weighting of 1,2, and 3 and a Hanning window with window sills $d k$ equal to 2 . Fits were performed in $R$ space over a $1.34 \AA$ range. As the initial model the structure of $\mathrm{LiNbO}_{3}$
(Ref. 12) was used. The amplitude reduction factor $\left(S_{0}^{2}\right)$ was held constant to 0.75 , the value obtained for initial fits for a Fe foil FT-EXAFS spectrum measured under the same experimental conditions. While fitting the FT-EXAFS spectra, the $S_{0}^{2}$ parameter was held constant, whereas $E_{0}\left(E_{0(\mathrm{O})}\right.$ and $\left.E_{0(\mathrm{Nb})}\right)$ and the Debye-Waller factors $\left(\sigma_{\mathrm{O}}^{2}\right.$ and $\left.\sigma_{\mathrm{Nb}}^{2}\right)$ were varied. The first coordination shell $(\mathrm{Fe}-\mathrm{O})$ was initially fitted by holding the coordination number constant to the model and varying the distance. Then, the coordination number was varied while holding the distance obtained in the initial fit constant. In this way the number of variables was kept 3 for 5.9 independent data points. The number of independent data points are calculated as

$$
N_{\text {ind }} \sim 2 \Delta R \Delta k / \pi,
$$

where $\Delta R$ and $\Delta k$ are the range widths of the analysis in $R$ and $k$ spaces, respectively.

In the next step the coordination number and the distance for the first coordination shell were held constant at their values obtained in the first step, and the fit range was extended to $3.4 \AA$, in order to include the second coordination shell $(\mathrm{Fe}-\mathrm{Nb})$. The fit procedure was repeated using two $\mathrm{Fe}-\mathrm{Nb}$ single scattering paths, but varying one $\Delta R_{\mathrm{Fe}-\mathrm{Nb}}$. The number of variables was 5 for 8.9 independent data points. For all performed fits, the obtained $\mathfrak{R}$-factor (overall goodness of fit) was 0.01 or better, which means that data and fit agree on average within $1 \%$ or better. $\mathfrak{R}$-factor is defined as

$$
\mathfrak{R}=\frac{\sum_{\mathrm{i}=1}^{N}\left\{\left[\operatorname{Re}\left(f_{i}\right)\right]^{2}+\left[\operatorname{Im}\left(f_{i}\right)\right]^{2}\right\}}{\sum_{\mathrm{i}=1}^{N}\left\{\left[\operatorname{Re}\left(\chi_{\text {data }_{i}}\right)\right]^{2}+\left[\operatorname{Im}\left(\chi_{\text {data }_{i}}\right)\right]^{2}\right\}},
$$

where $N=2\left(R_{\max }-R_{\min }\right) / \delta R+1$ is the number of points in the data range of the fit and $\delta R$ is the spacing in $R$ space. $f_{\mathrm{i}}$ is the difference between data and model in $R$ space defined as

$$
f_{i}\left(R_{i}\right)=\chi_{\text {data }}\left(R_{i}\right)-\chi_{\text {model }}\left(R_{i}\right) .
$$

The measurement of the absorption coefficient of the 2 wt $\%$ iron-doped $\mathrm{LiNbO}_{3}$ crystal was performed at $477 \mathrm{~nm}$ in transmission mode using a Cary 500 spectrometer in the VIS spectral range. Using the known relation ${ }^{3}$ between $\mathrm{Fe}^{2+}$ concentration $\left(c_{\mathrm{Fe}^{2+}}\right)$ and the absorption at $477 \mathrm{~nm}\left(\alpha_{477 \mathrm{~nm}}\right)$, $c_{\mathrm{Fe}^{2+}}=2.16 \times 1021 \mathrm{~m}^{-2} \alpha_{477 \mathrm{~nm}}$, the concentration of $\mathrm{Fe}^{2+}$ was calculated. An independent measurement by atomic absorption spectroscopy of the $\mathrm{LiNbO}_{3}: \mathrm{Fe} 2 \mathrm{wt} \%$ crystal leads to a total iron concentration of $1.11 \pm 0.03 \mathrm{wt} \%$.

\section{RESULTS AND DISCUSSIONS}

\section{A. EXAFS analyses}

The EXAFS spectra in $R$ and $k$ space of the $\mathrm{C} 1$ and $\mathrm{C} 2$ crystals are plotted along with their best fits in Fig. 1. The structural values obtained by fitting the intense peak at around $1.5 \AA$ (not phase corrected) suggest that the Fe atoms are sixfold coordinated by oxygen atoms in both samples (see Table I). Best fits in the $R$ range up to $3.4 \AA$ were achieved by employing a model with $\mathrm{Fe}$ atoms occupying $\mathrm{Li}$ sites, i.e., three $\mathrm{Nb}$ atoms located at around 3.05 and $3.14 \AA$ for the $\mathrm{C} 1$ and $\mathrm{C} 2$ crystals, respectively (see Table I). Fits 

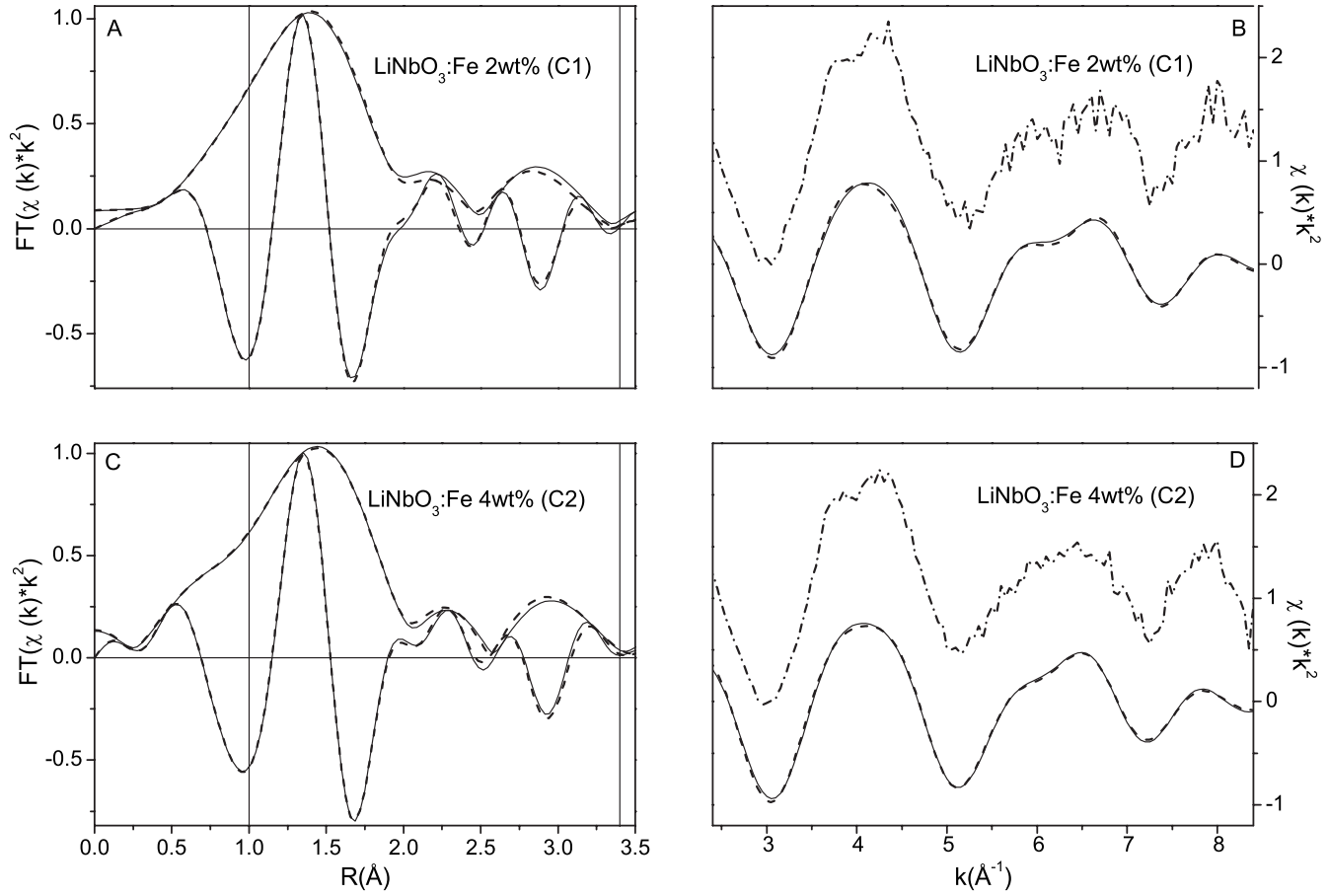

FIG. 1. EXAFS spectra of the [(a) and (b)] LN:Fe 2 wt $\%$ and [(c) and (d) $\mathrm{LN}: \mathrm{Fe} 4 \mathrm{wt} \%$ crystals in [(a) and (c)] $R$ (distance) (the magnitude and imaginary part) and [(b) and (d)] $k$ (wave number) [the experimental (dash dot) and Fourier filtered data (solid)] space and their best fits (dash).

using models of $\mathrm{Fe}_{2} \mathrm{O}_{3}$ clusters and incorporation of $\mathrm{Fe}$ atoms into $\mathrm{Li}$ and $\mathrm{Nb}$ sites yielded unsatisfactory results, which made us confident to rule them out. Results from the EXAFS analysis suggest that the iron atoms are dispersed in the LN:Fe 4 wt \% crystal. This contradicts the result of Olimov et al. ${ }^{7}$ who reported indications for formation of small iron oxide $\left(\mathrm{Fe}_{2} \mathrm{O}_{3}\right)$ clusters in a LN:Fe 4 wt \% crystal. The crystal studied in this paper and by Olimov et al. originates from the same crystal boule. The discrepancy might be due to the inhomogeneously distributed iron oxide in the boule. This scenario is likely because of the high doping level of iron. The significantly increased $\mathrm{Fe}-\mathrm{Nb}$ distance in the case of the $\mathrm{C} 2$ crystal, from 3.05 to $3.14 \AA$, provides evidence for distortion of the LN crystal matrix, which might be caused by a decreased distance between the Fe centers.

\section{B. XANES and VIS absorption analyses}

Figure 2 depicts the XANES spectra of the $\mathrm{C} 1$ and $\mathrm{C} 2$ crystals along with those of the $\mathrm{FeO}$ and $\mathrm{Fe}_{2} \mathrm{O}_{3}$ reference compounds. On the energy scale, the spectra of the two samples are positioned close to the spectrum of $\mathrm{Fe}_{2} \mathrm{O}_{3}$, which indicates a major presence of $\mathrm{Fe}^{3+}$ and a minor presence of $\mathrm{Fe}^{2+}$ in both crystals. This qualitative estimate is supported by the VIS absorption result, which found $20 \%$ of $\mathrm{Fe}^{2+}$ and $80 \%$ of $\mathrm{Fe}^{3+}$ in the 2 wt \% iron-doped LN crystal. The energy positions of the absorption edge of $\mathrm{Fe}$ in the two samples, determined as the maximum of the first derivative of the respective XANES spectrum, are identical. This finding implies similar $\mathrm{Fe}^{2+}$ to $\mathrm{Fe}^{3+}$ ratio in the $\mathrm{C} 2$ crystal.

The overall shape of a XANES spectrum reflects the electronic and geometrical structure of both $\mathrm{Fe}^{2+}$ and $\mathrm{Fe}^{3+}$ sites, which makes the extraction of site-selective structural information difficult. The in situ thermoelectric oxidization of $\mathrm{LN}: \mathrm{Fe} 2 \mathrm{wt} \%$ aimed not only to track the structural changes around the $\mathrm{Fe}$ atoms during the oxidation process but also to enable the assignment of spectral features to one of the two sites $\left(\mathrm{Fe}^{2+}\right.$ and $\left.\mathrm{Fe}^{3+}\right)$.

TABLE I. The results from the EXAFS analyses of the LN:Fe 2 wt \% (C1) and LN:Fe 4 wt \% (C2) spectra and the values of the structural parameters of the used Fe-into-the-Li-site model. The structural parameters are named as follows: $N$ denotes coordination numbers, $R(\AA)$ denotes distances, $\sigma^{2}\left(\AA^{2}\right)$ denotes Debye-Waller factors, and $\Delta E_{0}(\mathrm{eV})$ denotes energy shift of the ionization potential.

\begin{tabular}{|c|c|c|c|c|c|c|c|c|c|c|}
\hline & \multicolumn{4}{|c|}{$\begin{array}{c}\mathrm{LN}: \mathrm{Fe} \\
2 \mathrm{wt} \%(\mathrm{C} 1)\end{array}$} & \multicolumn{4}{|c|}{$\begin{array}{c}\mathrm{LN}: \mathrm{Fe} \\
4 \mathrm{wt} \%(\mathrm{C} 2)\end{array}$} & \multicolumn{2}{|c|}{$\begin{array}{c}\text { Used model: } \\
\text { Fe-into-the-Li site }\end{array}$} \\
\hline & $\begin{array}{l}R(\AA) \\
\pm 0.01\end{array}$ & $\mathrm{~N}$ & $\begin{array}{l}\sigma^{2}\left(\AA^{2}\right) \\
\pm 0.001\end{array}$ & $\Delta \mathbf{E}_{0}(\mathrm{eV})$ & $\begin{array}{l}R(\AA) \\
\pm 0.01\end{array}$ & $\mathrm{~N}$ & $\begin{array}{l}\sigma^{2}\left(\AA^{2}\right) \\
\pm 0.001\end{array}$ & $\Delta \mathbf{E}_{0}(\mathrm{eV})$ & $R(\AA)$ & $\mathrm{N}$ \\
\hline $\mathrm{O}^{1}$ & 1.98 & $6.6 \pm 0.5$ & 0.011 & $-3.4 \pm 0.4$ & 2.00 & $6.9 \pm 0.6$ & 0.012 & $-2.2 \pm 0.3$ & 2.07 & 3 \\
\hline $\mathrm{O}^{2}$ & & & & & & & & & 2.24 & 3 \\
\hline $\mathrm{Nb}^{1}$ & 3.01 & 1 & 0.011 & $-3.4 \pm 0.4$ & 3.10 & 1 & 0.012 & $2.0 \pm 0.9$ & 3.01 & 1 \\
\hline $\mathrm{Nb}^{2}$ & 3.05 & $3.3 \pm 0.6$ & 0.011 & $-3.4 \pm 0.4$ & 3.14 & $2.1 \pm 0.6$ & 0.012 & $2.0 \pm 0.9$ & 3.05 & 3 \\
\hline $\mathrm{O}^{3}$ & 3.28 & 3 & 0.011 & $-3.4 \pm 0.4$ & & & & & 3.29 & 3 \\
\hline
\end{tabular}




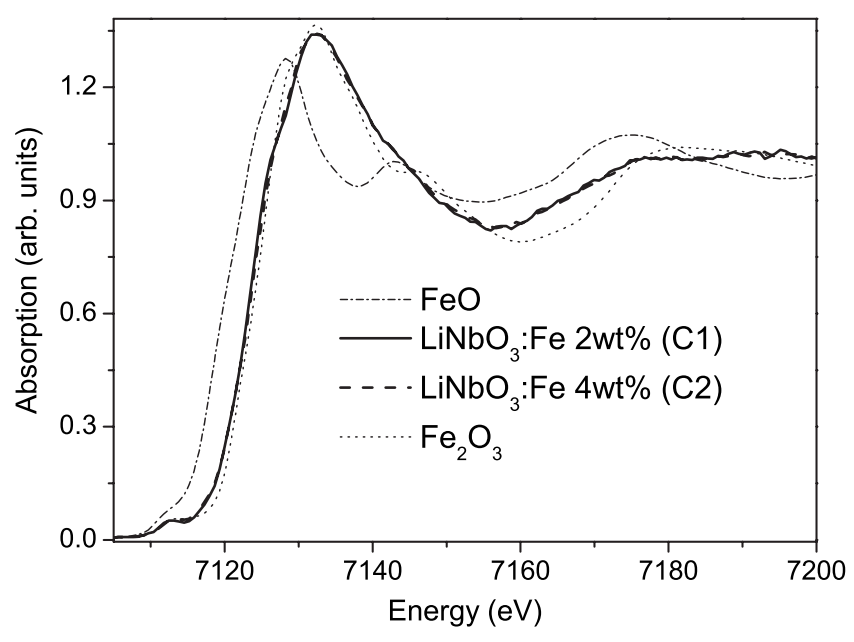

FIG. 2. Fe $K$-edge XANES experimental spectra of the LN:Fe 2 wt $\%$ and $\mathrm{LN}: \mathrm{Fe} 4 \mathrm{wt} \%$ crystals, and the $\mathrm{FeO}$ and $\mathrm{Fe}_{2} \mathrm{O}_{3}$ references.

\section{In situ studies}

During thermoelectric oxidization, iron-doped LN crystals change their color from completely black to completely transparent. ${ }^{5}$ This fingerprint was the first sign for successful oxidation of iron in the LN:Fe 2 wt \% crystal investigated in our in situ experiment. In Fig. 3, the XANES spectra, measured at the beginning ( $S_{-}$first) and at the end ( $S_{-}$last) of the oxidation treatment are shown. A $0.5 \mathrm{eV}$ energy shift of the S_last spectrum toward higher energies, compared to the S_first spectrum, was detected. The $0.5 \mathrm{eV}$ energy shift is measured as the difference in energy positions of the first inflection points of the two spectra, which are defined as the maximum of the first derivative of the respective XANES spectrum. The change in color and the energy shift of the spectrum toward higher energies indicate the successful oxidation of the crystal. However, the XANES spectrum does not show any other changes during the oxidation process, except this energy shift. Moreover, no significant differences were found in the measured EXAFS spectra. These findings suggest that both sites are most likely sixfold coordinated by oxygen atoms, and that the $\mathrm{Fe}^{2+}$ and $\mathrm{Fe}^{3+}$ atoms occupy the

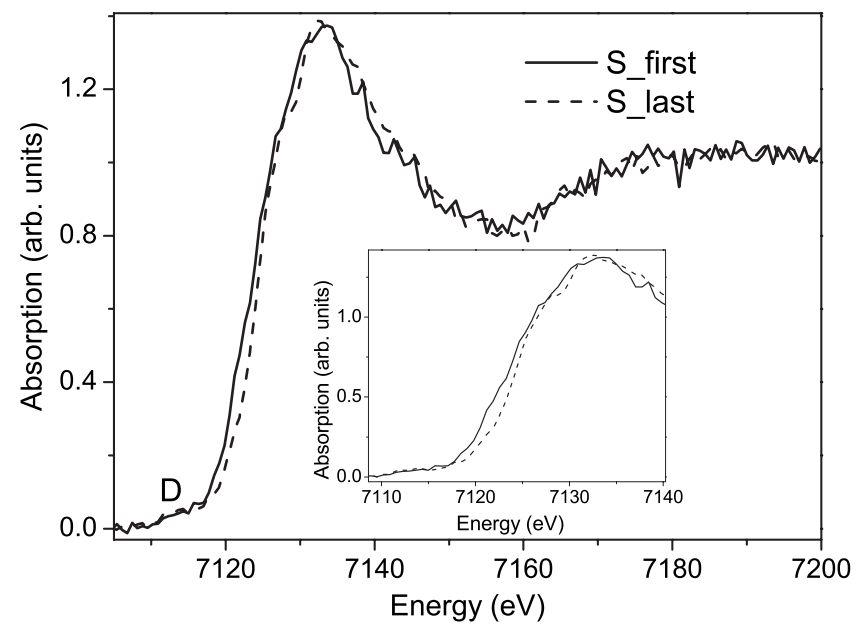

FIG. 3. The first (S_first) and the last (S_last) from the Fe $K$-edge XANES experimental spectra recorded during the in situ thermoelectric oxidization of a LN:Fe 2 wt \% crystal.

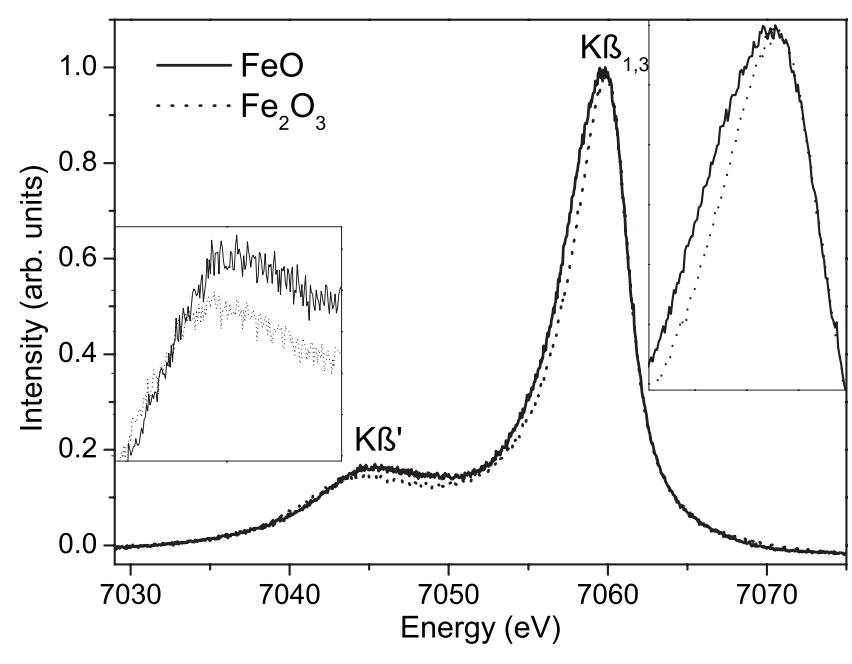

FIG. 4. The HRXE spectra of the $\mathrm{FeO}$ and $\mathrm{Fe}_{2} \mathrm{O}_{3}$ reference compounds. The insets are enlargements of the $K \beta^{\prime}$ and $K \beta$ lines.

Li site. Despite the similarities in short and long range geometrical structures, the electronic structure of the Fe atoms in the two valance states differs. The resonance at the pre-edge region of the S_first and S_last spectra [seen in Fig. 3(d)] is assigned to the transition of the $1 s$ electron to the lowest unoccupied states, and it is expected to reflect electronic structural changes. However, the low signal-to-noise ratio and the restricted resolution of a conventional XANES measurement prevent detection of such differences. In order to overcome these limitations, partially site-selective XANES spectra were extracted from a high-resolution RIXS map employing the chemical sensitivity of the high-resolution $K \beta$ emission lines of iron oxides with different oxidation states of iron.

\section{HRXE spectra and RIXS map}

The $1 s 3 p$ nonresonant HRXE spectra of $\mathrm{FeO}$ and $\mathrm{Fe}_{2} \mathrm{O}_{3}$ are displayed in Fig. 4. The $K \beta$ line is split into a main intense emission line $K \beta_{1,3}$, which reflects $3 p-1 s$ spin down transitions and a satellite $K \beta^{\prime}$ on the low energy site, which reflects $3 p-1 s$ spin-up transitions. The spin selectivity of the $K \beta$ emission line has been explained with the exchange interaction between the unpaired spin-up electrons in the $3 d$ state and the net $3 p$ electron spin after transition of a $3 p$ electron to the $1 s$ state. ${ }^{13-16}$ Since the energy difference between the two emission lines, the width of the $K \beta_{1,3}$ line, and the intensity of the $K \beta^{\prime}$ line depend on the number of spin-up electrons in the $3 d$ state, the $1 s 3 p$ HRXE spectra are sensitive to the oxidation state of iron (see Fig. 4). Using the chemical sensitivity of the $1 s 3 p$ emission lines, Glatzel et al. ${ }^{17}$ measured site-selective EXAFS spectra of a mixedvalence compound Prussian blue, which contains $\mathrm{Fe}^{2+}$ and $\mathrm{Fe}^{3+}$. By following this approach, we tried to extract siteselective XANES spectra, called A and B, from the RIXS map of the $\mathrm{C} 2$ crystal by fixing the emission energy at the low and high energy sides at half-maximum of the $K \beta_{1,3}$ emission line of the $\mathrm{C} 2$ crystal (see Figs. 5 and 6). Since the emission line of the $\mathrm{Fe}^{2+}$ reference $(\mathrm{FeO})$ is wider than that of the $\mathrm{Fe}^{3+}\left(\mathrm{Fe}_{2} \mathrm{O}_{3}\right)$ reference (Fig. 4), mainly $\mathrm{Fe}^{2+}$ is expected to contribute to the A XANES spectrum. As the $\mathrm{Fe}^{2+}$ 


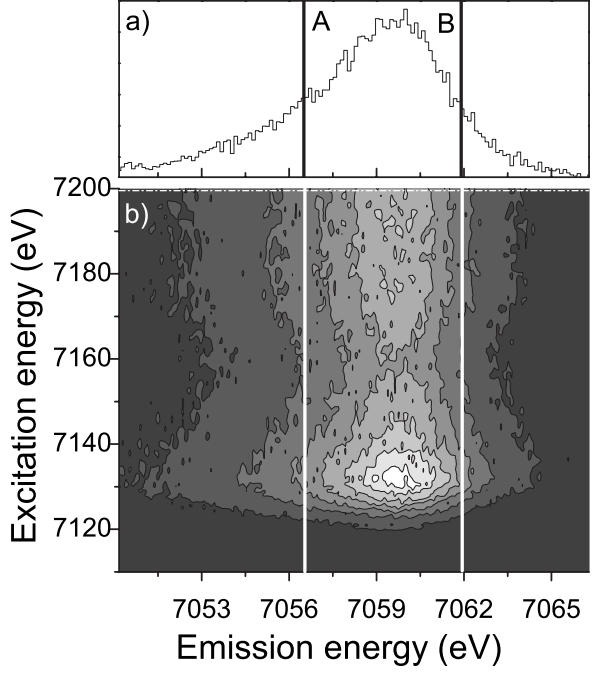

FIG. 5. (a) The $K \beta_{1,3}$ emission line and (b) the $1 s 3 p$ RIXS map of the $\mathrm{LN}: \mathrm{Fe} 4$ wt \% (C2) crystal.

and $\mathrm{Fe}^{3+}$ emission lines overlap, it is not possible to extract "pure" site-selective spectra with the available energy resolution. The A and B XANES spectra are plotted in Fig. 6. Despite the similarity of the two spectra, clear differences in the intensities and center of gravity of the two pre-edges are present (see the inset in Fig. 6). The two pre-edges are isolated by fitting a spline function and subtracting the remaining part of the spectra. The pre-edge resonances of the A and B spectra were modeled with Gauss profiles [see inset (a) in Fig. 6]. The Gauss profiles are not assigned to any atomic transitions. The aim of the fit is the determination of the center of gravity of the two structures. Wilke et al. ${ }^{18}$ showed in their systematic study of iron oxides that the center of gravity of the pre-edge feature for $\mathrm{Fe}^{2+}$ and $\mathrm{Fe}^{3+}$ compounds differs by $\approx 1.4 \mathrm{eV}$. This finding does not depend on the site symmetry of iron. The difference in center of gravity of the pre-edges of the $\mathrm{A}$ and $\mathrm{B}$ spectra is $\approx 1.4 \pm 0.1 \mathrm{eV}$. This result indicates that in the $\mathrm{A}$ and $\mathrm{B}$ spectra dominantly con-

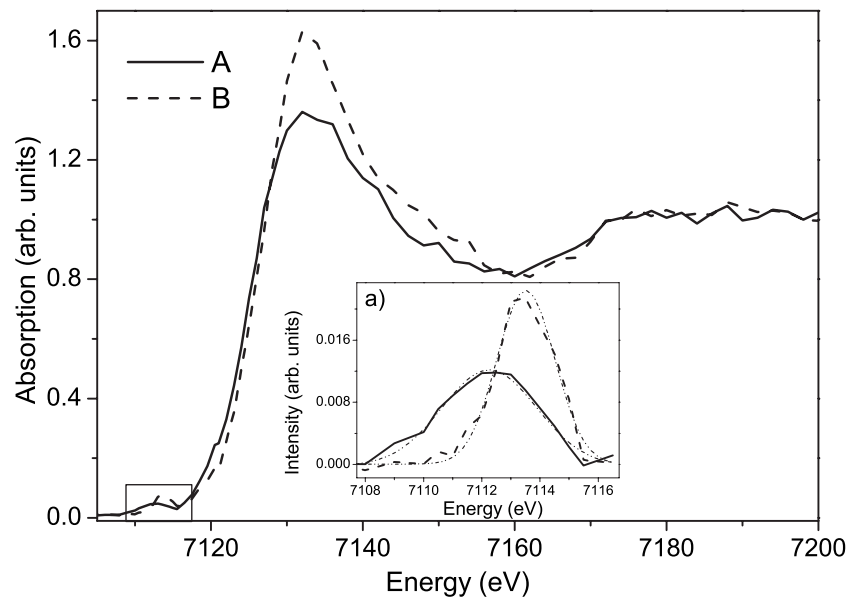

FIG. 6. Site-selective Fe $K$-edge XANES spectra A and B extracted from the RIXS map of the LN:Fe 4 wt \% crystal. The spectra A and B are obtained by integrating $\pm 1 \mathrm{eV}$ around the emission energies marked with $\mathrm{A}$ and B in [Fig. 5(a)], respectively. The background subtracted pre-edge resonances of the A and B spectra, their best fits, and the Gauss profiles (dash dot dot) used as models are shown in the inset (a). tribute $\mathrm{Fe}^{2+}$ and $\mathrm{Fe}^{3+}$, respectively. The similarity of the shape of the white lines and the multiple scattering regions of the two spectra suggest that the short range and long range geometrical structures of the two sites are similar. This finding agrees with the results obtained by the in situ experiment. However, in this specific case the partially site-selective XANES spectra are more sensitive, demonstrating the difference in the electronic structure of the $\mathrm{Fe}^{2+}$ and $\mathrm{Fe}^{3+}$ sites.

\section{CONCLUSION}

Based on XANES, EXAFS, VIS absorption studies, in situ measurements of thermoelectric oxidization of a $\mathrm{LN}: \mathrm{Fe}$ 2 wt \% crystal, and HRXE spectroscopic studies, we come to the following conclusions.

(1) The Fe atoms in the LN crystals doped with 2 and 4 wt $\%$ iron are sixfold coordinated by oxygen atoms and occupy Li sites. Formation of iron oxide clusters ( $\mathrm{Fe}-$ $\mathrm{O}-\mathrm{Fe}$ coordination) was not found within the sensitivity of the EXAFS analyses.

(2) It was possible to distinguish between the $\mathrm{Fe}^{2+}$ and $\mathrm{Fe}^{3+}$ sites and it was found that their site symmetries and positions in the $\mathrm{LN}$ matrix are very similar, i.e., the $\mathrm{Fe}^{2+}$ and $\mathrm{Fe}^{3+}$ atoms are in octahedral geometry surrounded by oxygen atoms and they both occupy the Li site.

\section{ACKNOWLEDGMENTS}

The authors gratefully acknowledge financial support for project B1 within the DFG research unit 557 . The support by Boris Brendebach, Jörg Rothe, and Kathy Dardenne at the INE beamline and Pawel Machek and Wolfgang Caliebe at the W1 beamline is highly appreciated. We thank Simo Huotari (ESRF) for providing the Si (531) analyzer crystal. We also thank the INE beamline team for critically reading the paper and giving valuable suggestions.

${ }^{1}$ P. Günter and J.-P. Huignard, Photorefractive Materials and Their Applications Springer, New York, 2005), Vol. 1; P. Günter and J.-P. Huignard, Photorefractive Materials and Their Applications (Springer, New York, 2006), Vol. 2; P. Günter and J.-P. Huignard, Photorefractive Materials and Their Applications (Springer, New York, 2007), Vol. 3.

${ }^{2}$ L. Arizmendi, Phys. Status Solidi A 201, 253 (2004).

${ }^{3}$ H. Kurz, E. Krätzig, W. Keened, H. Engelmann, U. Gonser, B. Discher, and A. Räuber, Appl. Phys. (Berlin) 12, 355 (1977).

${ }^{4}$ A. M. Glass, D. V. der Linde, and T. J. Negran, Appl. Phys. Lett. 25, 233 (1974).

${ }^{5}$ M. Falk and K. Buse, Appl. Phys. B: Lasers Opt. 81, 853 (2005).

${ }^{6}$ M. Falk, J. Japs, T. Woike, and K. Buse, Appl. Phys. B: Lasers Opt. 87, 119 (2007).

${ }^{7}$ K. Olimov, M. Falk, K. Buse, T. Woike, J. Hormes, and H. Modrow, J. Phys.: Condens. Matter 8, 5131 (2006).

${ }^{8}$ M. A. Denecke, J. Rothe, K. Dardenne, H. Blank, and J. Hormes, Phys. Scr., T 115, 1001 (2005)

${ }^{9}$ M. Lemonnier, O. Coller, C. Depautex, J. M. Esteva, and D. Raoux, Nucl. Instrum. Methods 152, 109 (1978).

${ }^{10}$ E. Welter, P. Machek, G. Dräger, U. Brüggmann, and M. Fröba, J. Synchrotron Radiat. 12, 448 (2005).

${ }^{11}$ B. Ravel and M. Newville, J. Synchrotron Radiat. 12, 537 (2005).

${ }^{12}$ ICSD No. 28294

${ }^{13}$ G. Peng, F. M. F. deGroot, K. Hämäläinen, J. A. Moore, X. Wang, M. M. Grush, J. B. Hastings, D. P. Siddons, W. H. Armstrong, O. C. Mullins, and S. P. Cramer, J. Am. Chem. Soc. 116, 2914 (1994).

${ }^{14}$ K. Tsutsumi, J. Phys. Soc. Jpn. 14, 1696 (1959). 
${ }^{15}$ A. Meisel, G. Leonhardt, and R. Szargan, in X-Ray Spectra and Chemical Binding, edited by F. P. Schäfer, V. I. Goldanskii, and J. P. Toennies, (Springer-Verlag, Berlin, 1989), Vol. 37.

${ }^{16}$ P. Glatzel and U. Bergmann, Coord. Chem. Rev. 249, 65 (2005).
${ }^{17}$ P. Glatzel, L. Jacquamet, U. Bergmann, F. M. F. de Groot, and S. P. Cramer, Inorg. Chem. 41, 3121 (2002).

${ }^{18}$ M. Wilke, F. Farges, P.-E. Petit, G. E. Brown, Jr., and F. Martin, Am. Mineral. 86, 714 (2001). 